\title{
Chemical hemorrhagic cystitis: Diagnostic and therapeutic pitfalls (Review)
}

\author{
RAZVAN-COSMIN PETCA ${ }^{1,2^{*}}$, RAZVAN-IONUT POPESCU ${ }^{1,2^{*}}$, CRISTIAN TOMA $^{1,2^{*}}$, \\ MIHAI CRISTIAN DUMITRASCU ${ }^{3,4}$, AIDA PETCA ${ }^{3,5}$, FLORICA SANDRU $^{6,7}$ and CALIN BOGDAN CHIBELEAN ${ }^{8,9}$
}

${ }^{1}$ Department of Urology, 'Carol Davila' University of Medicine and Pharmacy, 050474 Bucharest; ${ }^{2}$ Department of Urology, 'Prof. Dr. Th. Burghele' Clinical Hospital, 050659 Bucharest; ${ }^{3}$ Department of Obstetrics and Gynecology, 'Carol Davila' University of Medicine and Pharmacy, 050474 Bucharest; ${ }^{4}$ Department of Obstetrics and Gynecology,

University Emergency Hospital, 050098 Bucharest; ${ }^{5}$ Department of Obstetrics and Gynecology,

Elias Emergency University Hospital, 011461 Bucharest; ${ }^{6}$ Department of Dermatology,

'Carol Davila' University of Medicine and Pharmacy, 050474 Bucharest; ${ }^{7}$ Department of Dermatology,

Elias Emergency University Hospital, 011461 Bucharest; ${ }^{8}$ Department of Urology,

'George Emil Palade' University of Medicine, Pharmacy, Science and Technology of Targu-Mures,

540139 Targu-Mures; ${ }^{9}$ Department of Urology, Mureș County Hospital, 540136 Targu-Mures, Romania

Received February 19, 2021; Accepted March 23, 2021

DOI: $10.3892 /$ etm.2021.10056

\begin{abstract}
Chemical cystitis (CC) is an inflammation of the bladder caused by various chemical agents ingested intentionally or accidentally. It is linked to chemotherapeutic agents such as cyclophosphamide, therapeutic agents for diverse diseases, and anesthetic agents consumed abusively for recreational effects such as ketamine, or can be linked to environmental and surrounding factors such as soaps, gels, spermicides, and dyes. $\mathrm{CC}$ is a pathology with an increasing incidence that is inadequately treated due to its infectious cystitis-like symptoms. The hemorrhagic form can have a rampant evolution. Treatment options of $\mathrm{CC}$ and its complications are under continuous research with no accepted standardized sequence. In many situations, the treatments are difficult to obtain, administer, and follow-up. In addition, the lack of experience of the physician may pose other obstacles in delivering treatment to the patient. In conclusion, $\mathrm{CC}$ is a disease with an increasing incidence, challenging to diagnose, which is frequently mistreated, and has multiple treatment modalities that still require standardization in administration and sequencing.
\end{abstract}

Correspondence to: Dr Mihai Cristian Dumitrascu, Department of Obstetrics and Gynecology, University Emergency Hospital, 169 Splaiul Independentei, 050098 Bucharest, Romania

E-mail:drdumitrascu@yahoo.com

*Contributed equally

Key words: chemical cystitis, hemorrhagic cystitis, urinary bladder inflammation, noninfectious cystitis, hematuria, intravesical therapy

\section{Contents}

1. Introduction

2. Etiology

3. Diagnostic strategy

4. Differential diagnosis

5. Prevention

6. Treatment

7. Experimental and other agents

8. Conclusions

\section{Introduction}

The term cystitis is used to define general inflammation of the bladder (1). The inflammation can be chronic or acute, with the severity ranging from mild discomfort in the suprapubic region to significant hemorrhage, which can be life-threatening (2). For a more straightforward classification of the types of cystitis, they can be divided into two main categories: Infectious and noninfectious (2). According to their etiology, cystitis can occur secondary to radiation therapy, infection, chemical, or mechanical aggression, either as a part of interstitial cystitis or chronic pelvic pain syndrome (1-3). Many other pathologies mimic cystitis (4).

Chemical cystitis (CC) is quite often mistaken for hemorrhagic cystitis, which is, in fact, a complication of this disease (4). CC is caused by different chemical agents ingested orally, intravenously, through instillation therapy, or topical contiguity (2). CC is attributed to chemotherapeutic or immunologic drug administration such as cyclophosphamide $(5,6)$, medication administered for different pathologies $(2,7)$, or the use of certain anesthetic drugs in a recreational manner, such as ketamine (8). Direct contact of the female urethra with various gels, soaps, or spermicides, creating a rather allergenic-chemical reaction, can cause ascending inflammation of the bladder (2). 
CC shares similar symptoms with other types of cystitis, presenting with suprapubic pain, dysuria, urinary frequency, urinary urgency, and hematuria (microscopic or macroscopic). Hemorrhagic $\mathrm{CC}$ is one of the most challenging diseases to treat and can rapidly intensify to the necessity for cystectomy with urinary diversion $(9,10)$.

Hemorrhagic CC is similar to hemorrhagic radiation cystitis. Although the etiology and pathophysiology are quite different, the literature reports them together. Hemorrhagic $\mathrm{CC}$ is characterized by its sterility, even though this is not an exclusion criterion (3). There are clinical settings that describe hemorrhagic CC coexisting with infectious cystitis and vice versa when infectious cystitis becomes hemorrhagic in the context of a chemical agent inductor. Hemorrhagic CC can be caused by treatment with the chemotherapy drug, cyclophosphamide, and similar agents from its class $(3,11)$, by intravesical instillation therapy with bacillus Calmette-Guerin $(12,13)$, other therapeutic agents such as tiaprofenic acid (used to treat arthritic pain) $(7,14)$, or environmental chemical agents (15).

In the USA, in 2017, an estimated 712,614 individuals were living with bladder cancer. Evaluation of the cases and deaths reported between 2013 and 2017, it was shown that almost 2.4\% of people would be diagnosed with bladder cancer during their lifetime (16). A considerable amount of these people require administration of intravesical therapy and therefore are at risk for developing CC.

Ketamine is another risk factor for $\mathrm{CC}$, which is used on a large scale. For example, only in the USA, the National Survey on Drug Use and Health reported an estimated 2.3 million people aged over 12 who have used ketamine in their lifetime, with about 203,000 users in 2013 (8). Approximately one-third of long-term ketamine users develop ketamine-induced CC. Some European countries, along with Taiwan, Hong Kong, Singapore, and Malaysia, report rising a incidence of this complication of ketamine use. However, the actual prevalence of CC due to ketamine use is unknown (17).

Cyclophosphamide, which is used to treat vasculitis such as Wegener granulomatosis or malignant disease, can cause $\mathrm{CC}$ and also hemorrhagic CC (18). One study reported an incidence of $20-25 \%$ in patients who develop hemorrhagic CC after high-doses of cyclophosphamide (11). Another drug that has also been reported to cause hemorrhagic chemical cystitis is methotrexate, which is used at a low dose to treat rheumatoid arthritis (19).

Considering these statistics and incidence rates, we can state that $\mathrm{CC}$ is a serious, and potentially highly morbid disease that should be identified by all physicians. CC still needs standardization in regards to its diagnosis, treatment, follow-up, and complication management. Misdiagnosis and inadequate treatment are two of the main drawbacks of CC management. Even when physicians manage to establish a proper diagnosis, they are impeded by the lack of standardization and availability of treatment options. In addition, the lack of experience and adequate studies result in the hesitation of physicians to initiate treatment, as there is a misgiving of its efficacy and fear of the high risk of side effects.

Since the hemorrhagic complication is one of the most difficult to handle, we have decided to approach this subject thoroughly.

\section{Etiology}

$\mathrm{CC}$ is noninfectious cystitis, and it is caused by chemical irritation of the bladder mucosa. CC tends to be more aggressive than infectious cystitis, with more severe irritative lower urinary tract symptoms (LUTS), hematuria, and bladder pain (2). CC may be caused by several classes of therapeutic or diagnostic agents, but it is commonly identified after chemotherapeutic treatment with cyclophosphamide and iphosphamide (4).

Cyclophosphamide and iphosphamide. These medications are part of the therapeutic strategy used for B cell malignant disease and various solid tumors. They are integrated into the treatment protocol before bone marrow transplantation and for immunoinflammatory conditions (rheumatoid arthritis, granulomatosis of Wegener, systemic lupus) $(2,5,9)$.

Cyclophosphamide is an oxazophosphorine compound, and its urinary tract toxicity is proven to be dose-related (9). Both cyclophosphamide and iphosphamide, express a corrosive liver product called acrolein, which is filtered by the kidneys and consequently accumulates in the bladder. Acrolein induces a reaction with pyroptotic effects in the urothelium resulting in ulceration and exposure of the muscularis mucosa and the blood vessels. Another source of acrolein exposure is cigarette smoke and charred animal proteins (4). Acrolein can break down proteins, including DNA, which results in a metabolite that causes apoptosis (6). A mouse model study demonstrated that cyclophosphamide-induced CC caused necrosis of urothelial cells $(6,20,21)$.

Bacillus Calmette Guerin-bladder instillation therapy. The intravesical instillation with bacillus Calmette-Guerin (BCG) is the most effective prophylactic immunotherapy for intermediate or high-risk non-muscle-invasive bladder cancer, after transurethral resection of bladder tumors $(22,23)$. It is considered that a breach in the glycosaminoglycans GAG layer, which covers the urothelium in the bladder, is the first step of the CC physiopathologic mechanism (24). BCG instillation therapy can result in urothelial GAG loss (25). Although effective on bladder cancer recurrence, BCG therapy can cause severe systemic and topical side effects (26). More frequent local side effects are bladder irritation (difficult/frequent/bloody/painful urination), incontinence, groin, or bladder pain (26). Flu-like symptoms (weakness, aches, fever, chills), nausea, vomiting, constipation/diarrhea, headache, and skin rash are the most prevalent general side effects of BCG therapy $(23,26)$.

Brausi et al studied the correlation between the amount and frequency of BCG intravesical therapy and the prevalence and severity of CC (26). The research showed that $62.8 \%$ of the 1,316 patients developed local side effects, and $69.5 \%$ had both local and systemic symptoms. The most common local adverse effect was CC in approximately $35 \%$ of cases (460 patients out of 1,316). The survey comprised 4 study groups. It compared the one-third dose to full-dose effects and one year with three years of BCG therapy continuance. They observed no difference in side effects depending on the dose or duration of BCG treatment (26).

Mitomycin C. Mitomycin can be administered intravenously to treat upper gastrointestinal (GI) cancers such as esophageal and anal cancer, and also for breast cancer. In patients treated 
with mitomycin, CC with hemorrhagic manifestation or LUTS or both was associated with an incidence of 1 to $10 \%$ (27).

Mitomycin is also a type of intravesical instillation therapy administered for non-muscle-invasive bladder cancer. The incidence of $\mathrm{CC}$ with hemorrhagic or irritative symptoms is variable among several studies. One study by Filson et al surveyed the complications associated with single-dose perioperative mitomycin $\mathrm{C}$ for patients undergoing bladder tumor resection. Two groups were compared: One receiving perioperative mitomycin $\mathrm{C}$ after bladder resection and the other with endoscopic treatment alone. The complication rate was higher in the mitomycin $\mathrm{C}$ group. Out of 116 patients who received perioperative mitomycin $\mathrm{C}, 6$ had major complications described as CC. Five patients required cystoscopic evaluation or intervention-coagulation of the active hemorrhagic lesions, or both. One patient required radical cystectomy with an ileal conduit (28). Other studies report rates of $\mathrm{CC}$ related to mitomycin $\mathrm{C}$ ranging between $3-4 \%$ (29).

Ketamine abuse for recreational use. Ketamine hydrochloride, an anesthetic drug, is an N-methyl-D-aspartic receptor antagonist with rapid onset and short duration of action. Ketamine can be taken orally or snorted to obtain a recreational effect. Ketamine abusers still represent a significant number, spread among all socio-culture classes. The first proof of illegal abuse of ketamine was found on the western coast of the USA in the 70s (30). In 2000, individuals aged 12 to 25 accounted for $74 \%$ of the ketamine-related emergency department visits in the USA alone (15).

Bladder dysfunction among recreational ketamine abusers was reported for the first time by Shahani et al in a series of 9 cases of ulcerative cystitis in 2007 (31). Also called 'a murderer of young bladders', ketamine-associated cystitis, a type of CC, is known to develop in frequent users. Chronic ketamine use has been observed to cause ulcerative cystitis with symptoms ranging from hematuria, polyuria, urinary urgency, dysuria, nocturia, bladder pain, and decreased bladder capacity. With pathogenesis still unclear, literature data suggest the involvement of norketamine and hydroxynorketamine, two metabolites of ketamine (32).

Ketamine-induced CC generates more severe LUTS and smaller bladder capacity, conditions that are even more severe than bladder pain syndrome or interstitial cystitis (33).

Gentian violet, methylene blue, soaps, intimate gels, spermicides and other agents. Several case reports are described in the literature, which assigns different substances placed near or into the urethral meatus to cause inflammation of the urethra and also a type of allergenic-chemical reaction to the bladder. Due to the lack of data, there are no precise numbers concerning the prevalence and, nonetheless, about the pathophysiology or other aspects.

Gentian violet, used in the medical environment, is described more frequently as a cause of CC. CC was observed in a pediatric patient after the instillation of diluted gentian violet to assess bladder injury after herniorrhaphy; $\mathrm{CC}$ in this specific case evolved with gross hematuria (34). A woman accidentally introduced diluted gentian violet into the urethra instead of her vagina, as recommended, with the consequent development of dysuria, increased frequency, and burning pain in the lower abdomen (35).

\section{Diagnostic strategy}

Thompson et al have been working on a set of guidelines for chemical and radiation-induced cystitis since 2013, and their research has been an appreciated reference since then (2). The recommended diagnostic workup is not very different from a typical urological investigation path covering logical and affordable step-by-step strategy.

$\mathrm{CC}$ should be assessed with proper patient history, physical examination (especially the urogenital area), urinalysis, and urine culture. Cystoscopy under anesthesia, urinary cytology, and advanced imaging such as CT scans or MRI are usually required.

Essential aspects remain clinical history and physical examination. Patients with $\mathrm{CC}$ may present with different levels of hematuria, varying from microscopic to gross hematuria with clots. All lower urinary tract symptoms should be taken into consideration (2). Personal medical history is the key element in directing the final diagnosis towards CC. The physical examination must include abdominal inspection and palpation, respectively, a proper urogenital evaluation including, if possible, rectal, vaginal taxis, or both.

Urinalysis and urine culture are required to exclude infectious cystitis (36). In spite of its noninfectious feature, CC can coexist with infectious cystitis. The primary workup should include blood count, hemoglobin level, urea, creatinine, and coagulation profile.

As both European and American guidelines suggest, hematuria of unknown cause should be evaluated cystoscopically. Hemorrhagic CC can be evaluated cystoscopically under anesthesia with the possibility of taking a biopsy and also applying endoscopic treatment if appropriate. Thus, cystoscopy can be realized with both diagnostic and treatment purposes. Hydrodistension of the bladder for symptomatic relief of urinary urgency and polyuria is a method in hand (2).

Cytology should be considered, especially in patients with risk factors for urothelial carcinoma.

When faced with hematuria or with patients with a history of other pelvic malignancy (uterine carcinoma), imaging studies should be performed to assess the upper urinary tract and pelvis. MRI scan and CT urogram are suggested.

\section{Differential diagnosis}

$\mathrm{CC}$ and its complicated forms share similar symptoms with other types of cystitis. Lower urinary tract symptoms such as dysuria, from mild to severe, urinary frequency, urinary urgency, hematuria, and suprapubic pain are found in all types of cystitis (2). The diagnostic of CC is challenging and based on a rigorous medical history and physical examination.

$\mathrm{CC}$ can be misdiagnosed with all types of infectious (bacterial, fungal, viral, parasites) and noninfectious cystitis (radiation, interstitial, autoimmune).

All other pelvic and lower abdominal pathologies can be taken into consideration when the symptoms of CC are not very specific (for example, suprapubic or lower abdominal pain). Gynecology (uterine myomas, endometrial polyps, dysfunctional bleeding) or gastroenterology (hemorrhagic rectocolitis, internal hemorrhoids) conditions should be ruled out. 


\section{Prevention}

Benjamin Franklin said that an 'ounce of prevention is worth a pound of cure'. CC and its hemorrhagic form might be prevented by applying various affordable treatments.

Hyperhydration. Hyperhydration may be an accessible, affordable, and easy to apply treatment. The mechanism is speculated to consist of diuresis stimulation followed by the dilution of the aggressive chemical compounds in the urinary tract and the reduction in the quantity and the amount of time of contact with the urothelium. The published data sustain hyperhydration as a prophylactic tool to prevent $\mathrm{CC}$ in bone marrow transplant patients who receive cyclophosphamide. A study on more than 600 patients with stem cell transplant who received hyperhydration treatment and accelerated diuresis strategy showed a low incidence of hemorrhagic CC and lower grade 3-4 rates of this pathology (37). Grade 3 is defined as macroscopic hematuria with small clots, and grade 4-gross hematuria with clots causing urinary tract obstruction requiring instrumentation for clot evacuation (38).

Cranberry juice. Cranberry juice is probably one of the most commonly used phytotherapeutic agents in the prevention and treatment of lower urinary tract disorders $(39,40)$. D-mannose and proanthocyanidins, the two most studied components of cranberry fruits (41), may play a role in preventing CC. Several studies were realized in hemorrhagic radiation cystitis (42-44). Still, due to the results obtained on these patients and the protective effect on the bladder coating, some experts suggest using it as a prevention therapy (45).

Sodium 2-mercaptoethanesulphonate (MESNA). MESNA is a chemical compound designed to bind acrolein (the metabolite resulting from cyclophosphamide). It has been used as a prophylactic tool during chemotherapy and stem cell transplantation with variable results and is well-tolerated by patients (2).

Postprocedural bladder irrigation. Another treatment proposed to prevent $\mathrm{CC}$ and its complications consists of postprocedural bladder irrigation. Even though the available articles and data are contradicting, there are some good results. In a group of patients receiving an association of busulfan and cyclophosphamide after bone marrow transplantation, a lower incidence of hemorrhagic CC was recorded (46). Still, some aspects discourage the use of continuous bladder irrigation after high-dose cyclophosphamide treatment, such as patient dissatisfaction regarding the presence of a urinary catheter, the risk of UTI or urethral trauma, and other local side effects $(47,48)$. Some studies compare MESNA and continuous bladder irrigation and show that both of these methods decrease the risk of $\mathrm{CC}$ after cyclophosphamide treatment with no significant difference between the two preventative modalities (49).

\section{Treatment}

$\mathrm{CC}$ as stated before, can be accompanied by a wide range of symptoms, from diffuse suprapubic pain, dysuria, increased urinary frequency, urinary urgency to more severe symptoms such as fever or hematuria. The severity of the symptoms is difficult to analyze, especially if they are subjective ones such as urgency or dysuria. One of the symptoms of $\mathrm{CC}$, which can be classified by objective criteria, is hematuria. Hemorrhagic $\mathrm{CC}$ is classified by Droller et al into four main categories (38): i) Non-visible hematuria; ii) macroscopic hematuria; iii) macroscopic hematuria with small clots; and iv) gross hematuria with clots which cause urinary tract obstruction and require evacuation instrumentation.

Depending on the symptoms and their severity, the treatment for $\mathrm{CC}$ is patient-oriented. $\mathrm{CC}$ can be treated either symptomatically, with conservative therapy, or interventionally/surgically, with cystectomy and urinary diversion in patients with a more dramatic evolution.

Conservative therapy. Conservative therapy is a tool for (+/- mild) forms, presenting with dysuria, urinary frequency, and lower abdominal pain, yielding symptomatic treatment such as analgesic agents, spasmolytic, and anti-inflammatory medication (50). If hemorrhagic CC is suspected, grades Droller I-II or even III, intravenous hyperhydration can be considered (2). Patients who receive this therapy should be carefully assessed and monitored, especially if other cardio-respiratory pathologies or renal impairment diseases are associated $(51,52)$. Even though tranexamic acid was mostly investigated in the treatment of hemorrhagic radiation cystitis, there are recent findings that suggest it can be used to control the bleeding of various causes. Research on diverse forms of hematuria showed decreased bleeding time on continuous bladder instillation with saline (53).

Intravesical therapy. Intravesical therapy is another option used to treat $\mathrm{CC}$. The complicated hemorrhagic form of CC can be treated with many kinds of instillation therapies. In CC patients with high grade III or IV, the physician should first attempt to evacuate the bladder clots to maximize efficiency (10). The evacuation of the clots can be done through a catheter or by cystoscopic instrumentation. Intravesical therapy should be started with standard saline irrigation (both prevention and treatment), and followed by using agents such as sodium hyaluronate, chondroitin sulphate, prostaglandin, formalin, alum irrigation, and combinations of them.

Alum irrigation. Alum irrigation is an agent described for the management of chemical-induced hemorrhagic cystitis (2). Alum is a hydrated double sulfate of aluminum and potassium, which has astringent characteristics determining protein precipitation over bleeding surfaces. It is administered through a three-way catheter similar to the majority of intravesical therapies. The concentration should be $1 \%$, and the administration rate should not overpass $300 \mathrm{cc} / \mathrm{h}$ (54). Alum has been proven to be relatively safe to apply, with bladder spasms as the most common side effect, without signs of clinically significant systemic absorption. Intravesical alum therapy was found to offer a case resolution of hemorrhagic cystitis in approximately $60 \%$ of patients (55). Still, the literature supports alum to be an efficient way to control intractable hematuria in $\mathrm{CC}$, if no other treatment shows success $(55,56)$.

Formalin solution. Formalin solution is formaldehyde combined with water. Intravesical therapy with formalin for 
hemorrhagic CC is one of the most controversial methods. This therapy comes with significant possible complications ranging from fever, tachycardia, and hematuria to anuria, vesicovaginal or rectovaginal fistula, and even death $(57,58)$.

Chondroitin sulfate. Chondroitin sulfate is one of the more recently used therapies, attempting to stop hemorrhagic cystitis. The data are limited, and findings are focused mainly on curing or ameliorating chronic cystitis or radiation cystitis symptoms (2). Chondroitin sulfate acts locally by initiating and entertaining a urothelial coating, a biofilm formation, thus repairing the damage of the glycosaminoglycan barrier of the bladder epithelium.

Prostaglandins. Prostaglandins are lipid-derived autocoids produced by the sequential metabolism of arachidonic acid that sustain homeostatic functions and mediate pathological mechanisms, including inflammation (59). Prostaglandins may be useful to treat the hemorrhagic form of $\mathrm{CC}$ induced by cyclophosphamide therapy, according to various reports (60-62).

Sodium hyaluronate. Sodium hyaluronate is currently used for bladder pain syndrome or interstitial cystitis, which have similar symptoms as CC. Sodium hyaluronate was introduced in the treatment of both radiation and chemical-induced cystitis and showed a better response in patients with a chemical cause. Symptoms such as dysuria and pain from chemical and radiation cystitis were solved for $97 \%$ of patients as reported in a previous study (63).

Combination therapy. The combination therapy between hyaluronic acid and chondroitin sulfate has been proposed for the treatment of refractory BCG-induced CC. It showed significant and durable amelioration of suprapubic pain, urinary urgency, frequency, and increased urinary volume per voiding (64).

Systemic treatment. Systemic treatment addresses intractable hematuria with hyperbaric oxygen therapy, sodium pentosanpolysulphate, aminocaproic acid, hormones such as estrogen, and coagulation factor such as recombinant factor VIIa.

Hyperbaric oxygen therapy. Hyperbaric oxygen therapy with 20 - to 90 -min sessions of $100 \%$ oxygen inhalation at $0.3 \mathrm{MPa}$ in a hyperbaric chamber was found to be effective for cyclophosphamide-induced CC $(10,65,66)$. Increasing the tissue oxygen level as high as 15-fold, this method supports capillary angiogenesis and promotes the reformation of the damaged urothelium (65).

Sodium pentosanpolysulphate. Sodium pentosanpolysulphate administered to patients with hemorrhagic CC after chemotherapy or stem cell transplant reduced blood transfusion needs and mortality, with good tolerance $(9,67)$.

Aminocaproic acid. Aminocaproic acid, a synthetic lysine, diminishes fibrinolysis by competitively hindering plasminogen and plasmin (68). It is administered orally in doses going up to 100 to $150 \mathrm{mg} / \mathrm{kg}$ per day (69). Most of the drug is secreted unaffected in the urine. Events like thrombotic complications, myopathy, and rhabdomyolysis might occur during and after treatment $(69,70)$.

Conjugated estrogens. Systemic estrogen therapy is controversial as a strategy for CC treatment. Some older studies (71-73) report encouraging results for mild $\mathrm{CC}$ after oral administration of conjugated estrogens. Nevertheless, recent research (74) revealed poor response in the control group, at the expense of important adverse events, as venous thromboembolism and hormone-dependent malignancies.

Recombinant factor VIIa. Only a few and unconvincing data (75-78) are available so far about activated recombinant factor VIIa (NovoSeven) effect, infused intravenously, on patients with refractory $\mathrm{CC}$. The response is transitory, and all patients returned to the starting point level of $\mathrm{CC}$ within $24 \mathrm{~h}$ (75-77). Moreover, if bleeding is in the bladder as the primary site, NovoSeven treatment displayed a negative repercussion on the bleeding outcome (78).

\section{Experimental and other agents}

Anti-vascular endothelial growth factor (anti-VEGF). Anti-VEGF treatment has been observed in animal models to reduce bladder pain in cyclophosphamide-induced cystitis. The mechanism is based on the systemic blockade of VEGF signaling with anti-VEGF neutralizing antibodies (79).

Human choriogonadotropin (HCG). HCG was also assessed in an animal model with induced CC. The lowest interferon (INF) $-\gamma$ and tumor necrosis factor (TNF)- $\alpha$ were observed in the female rats with HCG treatment, suggesting the need for further investigation in this direction (80).

Urodynamics and histology studies observed on 37 Sprague-Dawley female rats with hydrochloric acid-induced $\mathrm{CC}$ found that tamoxifen citrate can be a treatment strategy for chronic inflammation and to improve bladder function (81).

There were also experimental studies that found no benefit in treating or controlling CC. Even though platelet-rich plasma is known to bring beneficial aspects in wound healing inflammatory responses, it seems that, used as an intravesical agent, it is not effective. Platelet-rich plasma used on 24 female rats with cyclophosphamide-induced CC did reverse the natural histopathological evolution of this type of bladder inflammation (82).

There are also human experimental studies with agents used for other pathologies, including bladder disease. Botulinum toxin A injection was shown to bring some benefits also to $\mathrm{CC}$ but on a small case series or case reports. Botulinum toxin A was first approved almost 10 years ago to treat neurogenic detrusor overactivity. It was then extended to refractory overactive bladder, detrusor sphincter dyssynergia with non-neurogenic dysfunctional voiding, or inappropriate relaxation of the urethra, interstitial cystitis, bladder pain syndrome, and chronic pelvic pain syndrome $(83,84)$. A small case series reported the benefit of intravesical botulinum toxin A injection with increased bladder capacity and diminished urinary frequency. The preliminary results suggest that injection of botulinum toxin A into the bladder can be used as a treatment for BCG-induced CC (85). 


\section{Conclusions}

$\mathrm{CC}$ is a formidable disease with an increasing incidence and exhibits signs and symptoms similar to other types of bladder inflammation disorders. Preventative methods or therapeutic approaches to reduce the symptoms and manifestation of possible CC are usually accessible and affordable (hyperhydration, continuous bladder irrigation, MESNA, cranberry juice). For now, the treatment consists mainly of symptomatic medication; the specific treatment still needs standardization, as some substances may be problematic through their side effects.

Ongoing research is needed to confirm more efficient treatment possibilities and new agents for favorable results in patients diagnosed with chemical cystitis.

\section{Acknowledgements}

Not applicable.

\section{Funding}

No funding was received.

\section{Availability of data and materials}

All information included in this review is documented by relevant references.

\section{Authors' contributions}

RCP, MCD and CBC designed the review. RIP, CT, AP and FS conducted the literature review, selected the literature findings and drafted the manuscript. RCP, MCD and CBC substantially contributed to the conception of the work, revised and edited the final manuscript. All authors read and approved the final version of the manuscript.

\section{Ethics approval and consent to participate}

Not applicable.

\section{Patient consent for publication}

Not applicable.

\section{Competing interests}

The authors declare that they have no competing interests.

\section{References}

1. Bonkat G, Bartoletti R, Bruyère F, Cai T, Geerlings SE, Köves B Schubert S, Wagenlehner F, Mezei T, Pilatz A, et al: EAU Guidelines on Urological Infections. EAU Guidelines Office, Arnhem, pp13, 2020.

2. Thompson A, Adamson A, Bahl A, Borwell J, Dodds D, Heath C, Huddart R, McMenemin R, Patel P, Peters J and Payne H: Guidelines for the diagnosis, prevention and management of chemical- and radiation-induced cystitis. J Clin Urol 7: 25-35, 2014

3. FrassettoLA,BreyerBN,KimED,GoluboffET,Leslie SW,Sant GR and Talavera F: Nonbacterial and noninfectious cystitis. Available from: https://emedicine.medscape.com/article/2056602-overview. Accessed May 302020.
4. Haldar S, Dru C and Bhowmick NA: Mechanisms of hemorrhagic cystitis. Am J Clin Exp Urol 2: 199-208, 2014.

5. Mukhtar S and Woodhouse C: The management of cyclophosphamide-induced haematuria. BJU Int 105: 908-912, 2010.

6. Korkmaz A, Topal T and Oter S: Pathophysiological aspects of cyclophosphamide and ifosfamide induced hemorrhagic cystitis; implication of reactive oxygen and nitrogen species as well as PARP activation. Cell Biol Toxicol 23: 303-312, 2007.

7. Crawford ML, Waller PC and Wood SM: Severe cystitis associated with tiaprofenic acid. Br J Urol 79: 578-584, 1997.

8. Addiction Center: Ketamine addiction and abuse. Available from https://www.addictioncenter.com/drugs/hallucinogens/ketamine/. Accessed May 30, 2020.

9. Manikandan R, Kumar S and Dorairajan LN: Hemorrhagic cystitis: A challenge to the urologist. Indian J Urol 26: 159-166, 2010.

10. Choong SK, Walkden M and Kirby R: The management of intractable haematuria. BJU Int 86: 951-959, 2000.

11. Emadi A, Jones RJ and Brodsky RA: Cyclophosphamide and cancer: Golden anniversary. Nat Rev Clin Oncol 6: 638-647, 2009.

12. Lamm D, Persad R, Colombel M and Brausi M: Maintenance bacillus Calmette-Guérin: The standard of care for the prophylaxis and management of intermediate- and high-risk non-muscle-invasive bladder cancer. Eur Urol Suppl 9: 715-734, 2010.

13. Hall MC, Chang SS, Dalbagni G, Pruthi RS, Seigne JD, Skinner EC, Wolf JS Jr and Schellhammer PF: Guideline for the management of nonmuscle invasive bladder cancer (stages Ta, T1, and Tis): 2007 update. J Urol 178: 2314-2330, 2007.

14. Buchbinder R, Forbes A, Kobben F, Boyd I, Snow RM and McNeil JJ: Clinical features of tiaprofenic acid (surgam) associated cystitis and a study of risk factors for its development. J Clin Epidemiol 53: 1013-1019, 2000.

15. Folland DS, Kimbrough RD, Cline RE, Swiggart RC and Schaffner W: Acute hemorrhagic cystitis: Industrial exposure to the pesticide chlordimeform. JAMA 239: 1052-1055, 1978.

16. National Cancer Institute: Cancer stat facts: Bladder cancer. Available from: www.seer.cancer.gov/statfacts/html/urinb.html. Accessed May 30, 2020.

17. Lee YK, Jhang JF and Kuo HC: Clinical outcome of augmentation enterocystoplasty for patients with ketamine-induced cystitis. Pain Physician 20: E431-E436, 2017.

18. Martin-Suarez I, D'Cruz D, Mansoor M, Fernandes AP, Khamashta MA and Hughes GR: Immunosuppressive treatment in severe connective tissue diseases: Effects of low dose intravenous cyclophosphamide. Ann Rheum Dis 56: 481-487, 1997.

19. Kawabe Y,Eguchi K, Tsuboi M, Kita M, Tsukada T, Takashima H, Mizokami A, Kawakami A, Matsuoka N, Migita K and Nagataki S: Untoward effects of low dose methotrexate therapy in rheumatoid arthritis. Ryumachi 36: 514-521, 1996 (In Japanese).

20. Juszczak K, Kaszuba-Zwoińska J, Chorobik P, Ziomber A and Thor PJ: The effect of hyperosmolar stimuli and cyclophosphamide on the culture of normal rat urothelial cells in vitro. Cell Mol Biol Lett 17: 196-205, 2012.

21. Zupancic D, Jezernik K and Vidmar G: Effect of melatonin on apoptosis, proliferation and differentiation of urothelial cells after cyclophosphamide treatment. J Pineal Res 44: 299-306, 2008.

22. Babjuk M, Oosterlinck W, Sylvester R, Kaasinen E, Böhle A, Palou-Redorta J and Rouprêt M; European Association of Urology (EAU): EAU guidelines on non-muscle-invasive urothelial carcinoma of the bladder, the 2011 update. Eur Urol 59: 997-1008, 2011.

23. Brausi M, Witjes JA, Lamm D, Persad R, Palou J, Colombel M, Buckley R, Soloway M, Akaza $\mathrm{H}$ and Böhle A: A review of current guidelines and best practice recommendations for the management of nonmuscle invasive bladder cancer by the international bladder cancer group. J Urol 186: 2158-2167, 2011.

24. Bassi PF, Costantini E, Foley S and Palea S: Glycosaminoglycan therapy for bladder diseases: Emerging new treatments. Eur Urol Suppl 10: 451-459, 2011

25. Lazzeri M and Montorsi F: The therapeutic challenge of "chronic cystitis': Search well, work together, and gain results. Eur Urol 60: 78-80, 2011.

26. Brausi M, Oddens J, Sylvester R, Bono A, van de Beek C, van Andel G, Gontero P, Turkeri L, Marreaud S, Collette S and Oosterlinck W: Side effects of Bacillus Calmette-Guérin (BCG) in the treatment of intermediate- and high-risk Ta, T1 papillary carcinoma of the bladder: Results of the EORTC genito-urinary cancers group randomised phase 3 study comparing one-third dose with full dose and 1 year with 3 years of maintenance BCG. Eur Urol 65: 69-76, 2014. 
27. Mitomycin Side Effects. Available from: https://www.drugs. $\mathrm{com} / \mathrm{sfx} / \mathrm{mitomycin}$-side-effects.html. Accessed May 30, 2020.

28. Filson CP, Montgomery JS, Dailey SM, Crossley HS, Lentz H, Tallman CT, He C and Weizer AZ: Complications associated with single-dose, perioperative mitomycin- $\mathrm{C}$ for patients undergoing bladder tumor resection. Urol Oncol 32: 40.e1-e8, 2014.

29. Solsona E, Iborra I, Ricós JV, Monrós JL, Casanova J and Dumont R: Effectiveness of a single immediate mitomycin C instillation in patients with low risk superficial bladder cancer: Short and long-term followup. J Urol 161: 1120-1123, 1999.

30. Jansen KL: A review of the nonmedical use of ketamine: Use, users and consequences. J Psychoactive Drugs 32: 419-433, 2000

31. Shahani R, Streutker C, Dickson B and Stewart RJ: Ketamineassociated ulcerative cystitis: A new clinical entity. Urology. 69 810-812. 2007.

32. Meng E, Wu ST, Cha TL, Sun GH, Yu DS and Chang SY: A murderer of young bladders: Ketamine-associated cystitis. Urol Sci 24: 113-116, 2013.

33. Chen WC, Lee MH, Lee SP, Chen YL, Wu HC, Lin HM and Liou WB: The patients with ketamine-induced cystitis (KIC) have more severe lower urinary tract symptoms and smaller bladder capacity than patients with interstitial cystitis/bladder painful syndrome (IC/BPS). Neurourol Urodyn 31: 1005-1006, 2012.

34. Kim SJ, Koh DH, Park JS, Ahn HS, Choi JB and Kim YS: Hemorrhagic cystitis due to intravesical instillation of gentian violet completely recovered with conservative therapy. Yonsei Med J 44: 163-165, 2003.

35. Magee P: Antiseptic drugs and disinfectants. In: Side Effects of Drugs Annual. 1st edition. Vol 23. Aronson JK (ed). Elsevier Science, Amsterrntam, pp247-249, 2000.

36. Petca RC, Mareș C, Petca A, Negoiță S, Popescu RI, Boț M, Barabás E and Chibelean CB: Spectrum and antibiotic resistance of uropathogens in Romanian females. Antibiotics (Basel) 9: 472, 2020.

37. Trotman J, Nivison-Smith I and Dodds A: Haemorrhagic cystitis: Incidence and risk factors in a transplant population using hyperhydration. Bone Marrow Transplant 23: 797-801, 1999.

38. Droller MJ, Saral R and Santos G: Prevention of cyclophosphamide-induced hemorrhagic cystitis. Urology 20: 256-258, 1982.

39. Jepson RG, Williams G and Craig JC: Cranberries for preventing urinary tract infections. Cochrane Database Syst Rev 10 CD001321 2012

40. Das S: Natural therapeutics for urinary tract infections-a review. Futur J Pharm Sci 6: 64, 2020

41. Sihra N, Goodman A, Zakri R, Sahai A and Malde S: Nonantibiotic prevention and management of recurrent urinary tract infection. Nat Rev Urol 15: 750-776, 2018

42. Cowan CC, Hutchison C, Cole T, Barry SJ, Paul J, Reed NS and Russell JM: A randomised double-blind placebo-controlled trial to determine the effect of cranberry juice on decreasing the incidence of urinary symptoms and urinary tract infections in patients undergoing radiotherapy for cancer of the bladder or cervix. Clin Oncol (R Coll Radiol) 24: e31-e38, 2012.

43. Campbell G, Pickles T and D'yachkova Y: A randomised trial of cranberry versus apple juice in the management of urinary symptoms during external beam radiation therapy for prostate cancer. Clin Oncol (R Coll Radiol) 15: 322-328, 2003.

44. Hamilton K, Bennett NC, Purdie G and Herst PM: Standardized cranberry capsules for radiation cystitis in prostate cancer patients in New Zealand: A randomized double blinded, placebo controlled pilot study. Support Care Cancer 23: 95-102, 2015

45. Herst PM, Aumata A, Sword V, Jones R, Purdie G and Costello S: Cranberry capsules are not superior to placebo capsules in managing acute non-haemorrhagic radiation cystitis in prostate cancer patients: A phase III double blinded randomised placebo controlled clinical trial. Radiother Oncol 149: 117-123, 2020.

46. Turkeri LN, Lum LG, Uberti JP, Abella E, Momin F, Karanes C, Sensenbrenner LL and Haas GP: Prevention of hemorrhagic cystitis following allogeneic bone marrow transplant preparative regimens with cyclophosphamide and busulfan: Role of continuous bladder irrigation. J Urol 153: 637-640, 1995.

47. Hensley ML, Hagerty KL, Kewalramani T, Green DM, Meropol NJ, Wasserman TH, Cohen GI, Emami B, Gradishar WJ, Mitchell RB, et al: American society of clinical oncology 2008 clinical practice guideline update: Use of chemotherapy and radiation therapy protectants. J Clin Oncol 27: 127-145, 2009.

48. Ballen KK, Becker P, Levebvre K, Emmons R, Lee K, Levy W, Stewart FM, Quesenberry P and Lowry P: Safety and cost of hyperhydration for the prevention of hemorrhagic cystitis in bone marrow transplant recipients. Oncology 57: 287-292, 1999.
49. Vose JM, Reed EC, Pippert GC, Anderson JR, Bierman PJ, Kessinger A, Spinolo J and Armitage JO: Mesna compared with continuous bladder irrigation as uroprotection during high-dose chemotherapy and transplantation: A randomized trial. J Clin Oncol 11: 1306-1310, 1993.

50. Tiglis M, Neagu TP, Elfara M, Diaconu CC, Bratu OG, Vacaroiu IA and Grintescu IM: Nefopam and its role in modulating acute and chronic pain. Rev Chim (Bucharest) 69: 2877-2880, 2018

51. Petca RC, Popescu RI, Mehedintu C, Bot M, Veduta A and Petca A: The role of ultrasound in diagnosis and management in renal colic during pregnancy. In: Proceedings of the 6th Congress of the Ultrasound Society in Obstetrics and Gynecology/34th Fetus as a Patient International Congress. Bucharest, pp478-484, 2018

52. Diaconu CC: Midaortic syndrome in a young man: Case presentation. Cor Vasa 59: e171-e173, 2017

53. Moharamzadeh P, Ojaghihaghighi S, Amjadi M, Rahmani F and Farjamnia A: Effect of tranexamic acid on gross hematuria: A pilot randomized clinical trial study. Am J Emerg Med 35: 1922-1925, 2017

54. Decker DB, Karam JA and Wilcox DT: Pediatric hemorrhagic cystitis. J Pediatr Urol 5: 254-264, 2009.

55. Westerman ME, Boorjian SA and Linder BJ: Safety and efficacy of intravesical alum for intractable hemorrhagic cystitis: A contemporary evaluation. Int Braz J Urol 42: 1144-1149, 2016.

56. Ho CC and Md Zainuddin Z: Alum irrigation for the treatment of intractable haematuria. Malays J Med Sci 16: 66-68, 2009.

57. Dewan AK, Mohan GM and Ravi R: Intravesical formalin for hemorrhagic cystitis following irradiation of cancer of the cervix. Int J Gynaecol Obstet 42: 131-135, 1993.

58. Vicente J, Rios G and Caffaratti J: Intravesical formalin for the treatment of massive hemorrhagic cystitis: Retrospective review of 25 cases. Eur Urol 18: 204-206, 1990.

59. Ricciotti E and FitzGerald GA: Prostaglandins and inflammation. Arterioscler Thromb Vasc Biol 31: 986-1000, 2011.

60. Demir HA, Savaş Şen Z, Altuğ MU, Emir S and Tunç B: Successful treatment of cyclophosphamide-induced haemorrhagic cystitis with intravesical prostaglandin E1 in a child with non-Hodgkin's lymphoma. Scand J Urol Nephrol 45: 281-284, 2011.

61. Yamamoto M, Hibi H, Ohmura M and Miyake K: Successful treatment of hemorrhagic cystitis secondary to cyclophosphamide chemotherapy with intravesical instillation of prostaglandin F2 alpha. Hinyokika Kiyo 40: 833-835, 1994.

62. Ippoliti C, Przepiorka D, Mehra R, Neumann J, Wood J, Claxton D, Gajewski J, Khouri I, van Besien K, Andersson B, et al: Intravesicular carboprost for the treatment of hemorrhagic cystitis after marrow transplantation. Urology 46: 811-815, 1995.

63. Sommariva ML, Sandri SD and Ceriani V: Efficacy of sodium hyaluronate in the management of chemical and radiation cystitis. Minerva Urol Nefrol 62: 145-150, 2010.

64. Imperatore V, Creta M, Di Meo S, Buonopane R, Longo N, Fusco F, Spirito L, Imbimbo C and Mirone V: Intravesical administration of combined hyaluronic acid and chondroitin sulfate can improve symptoms in patients with refractory bacillus Calmette-Guerin-induced chemical cystitis: Preliminary experience with one-year follow-up. Arch Ital Urol Androl 90: 11-14, 2018.

65. Davis M, MacDonald H, Sames C and Nand K: Severe cyclophosphamide-induced haemorrhagic cystitis treated with hyperbaric oxygen. N Z Med J 124: 48-54, 2011.

66. Ajith Kumar S, Prasanth P, Tripathi K and Ghosh P: Hyperbaric oxygen-a new horizon in treating cyclophosphamide-induced hemorrhagic cystitis. Indian J Urol 27: 272-273, 2011.

67. Duthie G, Whyte L, Chandran H, Lawson S, Velangi M and McCarthy L: Introduction of sodium pentosan polysulfate and avoidance of urethral catheterisation: Improved outcomes in children with haemorrhagic cystitis post stem cell transplant/chemotherapy. J Pediatr Surg 47: 375-379, 2012.

68. Abt D, Bywater M, Engeler DS and Schmid HP: Therapeutic options for intractable hematuria in advanced bladder cancer. Int J Urol 20: 651-660, 2013.

69. Stefanini M, English HA and Taylor AE: Safe and effective, prolonged administration of epsilon aminocaproic acid in bleeding from the urinary tract. J Urol 143: 559-561, 1990.

70. Kaye JD, Smith EA, Kirsch AJ, Cerwinka WH and Elmore JM: Preliminary experience with epsilon aminocaproic acid for treatment of intractable upper tract hematuria in children with hematological disorders. J Urol 184: 1152-1157, 2010. 
71. Liu YK, Harty JI, Steinbock GS, Holt HA Jr, Goldstein DH and Amin M: Treatment of radiation or cyclophosphamide induced hemorrhagic cystitis using conjugated estrogen. J Urol 144 41-43, 1990.

72. Miller J, Burfield GD and Moretti KL: Oral conjugated estrogen therapy for treatment of hemorrhagic cystitis. J Urol 151: 1348-1350, 1994.

73. Ordemann R, Naumann R, Geissler G, Bornhauser M, Schuler U and Ehninger G: Encouraging results in the treatment of haemorrhagic cystitis with estrogen-report of 10 cases and review of the literature. Bone Marrow Transplant 25: 981-985, 2000.

74. Mousavi SA, Moazed V, Mohebbi N, Hadjibabaie M, Alimoghaddam K, Bahar B, Jahani M and Ghavamzadeh A: Conjugated estrogen in late-onset hemorrhagic cystitis associated with hematopoietic stem cell transplantation. Int J Hematol Oncol Stem Cell Res 11: 13-18, 2017.

75. Geisler JP, Linnemeier GC and Manahan KJ: Recombinant factor VIIa to treat late radiation-induced hemorrhagic cystitis: A case report. J Reprod Med 53: 360-362, 2008.

76. Ashrani AA, Gabriel DA, Gajewski JL, Jacobs DR Jr, Weisdorf DJ and Key NS: Pilot study to test the efficacy and safety of activated recombinant factor VII (NovoSeven) in the treatment of refractory hemorrhagic cystitis following high-dose chemotherapy. Bone Marrow Transplant 38: 825-828, 2006.

77. Karimi M, Zakerinia M, Khojasteh HN, Ramzi M and Ahmad E: Successful treatment of cyclophosphamide induced intractable hemorrhagic cystitis with recombinant FVIIa (NovoSeven) after allogenic bone marrow transplantation. J Thromb Haemost 2: $1853-1855,2004$

78. Pihusch M, Bacigalupo A, Szer J, von Depka Prondzinski M, Gaspar-Blaudschun B, Hyveled L and Brenner B; F7BMT-1360 Trial Investigators: Recombinant activated factor VII in treatment of bleeding complications following hematopoietic stem cell transplantation. J Thromb Haemost 3: 1935-1944, 2005.
79. Lai HH, Shen B, Vijairania P,Zhang X, Vogt SK and Gereau RW IV: Anti-vascular endothelial growth factor treatment decreases bladder pain in cyclophosphamide cystitis: A multidisciplinary approach to the study of chronic pelvic pain (MAPP) research network animal model study. BJU Int 120: 576-583, 2017.

80. Tanik S, Zengin K, Albayrak S, Gurel A, Atar M, Sahin S, Tuzcu N, Tuzcu M, Imamoglu MA and Gurdal M: Assessment of therapeutic effect of human choriogonadotropin in a chemical cystitis model. Kaohsiung J Med Sci 33: 229-235, 2017.

81. Acar D, Cayan S, Aktaş S, Tek M and Akbay E: The effect of tamoxifen on bladder functions and histology, and the role of estrogen receptor beta in a rat chemical cystitis model. Neurourol Urodyn 26: 309-316, 2007.

82. Ozyuvali E, Yildirim ME, Yaman T, Kosem B, Atli O and Cimentepe E: Protective effect of intravesical platelet-rich plasma on cyclophosphamide-induced hemorrhagic cystitis. Clin Invest Med 39: 27514, 2016.

83. Jiang YH, Liao CH and Kuo HC: Current and potential urological applications of botulinum toxin A. Nat Rev Urol 12: 519-533, 2015.

84. Chibelean CB, Nechifor-Boilă IA, Petca RC, Balan D, Martha O, Petca A, and Dogaroiu C: Abobotulinumtoxin A in idiopathic hyperactive bladder-ethical issues. Rom J Leg Med 27: 213-216, 2019.

85. Chuang YC, Kim DK, Chiang PH and Chancellor MB: Bladder botulinum toxin A injection can benefit patients with radiation and chemical cystitis. BJU Int 102: 704-706, 2008. 